\title{
Stress intensity factors for functionally graded solid cylinders
}

\author{
Chunyu $\mathrm{Li}^{\mathrm{a}}{ }^{\text {** }}$, Zhenzhu Zou ${ }^{\mathrm{a}}$, Zhuping Duan ${ }^{\mathrm{b}}$ \\ a Shijiazhuang Railway Institute, Shijiazhuang 050043, People's Republic of China \\ ${ }^{\mathrm{b}}$ LNM, Institute of Mechanics, Chinese Academy of Sciences, People's Republic of China
}

Received 2 June 1998; received in revised form 1 February 1999; accepted 17 April 1999

\begin{abstract}
This paper presents the mode I stress intensity factors for functionally graded solid cylinders with an embedded penny-shaped crack or an external circumferential crack. The solid cylinders are assumed under remote uniform tension. The multiple isoparametric finite element method is used. Various types of functionally graded materials and different gradient compositions for each type are investigated. The results show that the material property distribution has a quite considerable influence on the stress intensity factors. The influence for embedded cracks is quite different from that for external cracks. (C) 1999 Elsevier Science Ltd. All rights reserved.
\end{abstract}

Keywords: Functionally graded materials; Stress intensity factor; Finite element method; Solid cylinder; Crack

\section{Introduction}

Solid cylinders have extensively practical applications in engineering. The problem of a solid cylinder with a crack, which is embedded or at the surface, has been treated by many researchers. However, these researches mainly concentrated on homogeneous materials. Recent years, developments in the space, nuclear, and chemical industries have placed new demands on materials suitable for unusual conditions of pressure, temperature, and environment. The concept of so-called Functionally Gradient Materials (FGMs) has been introduced and applied to the development of structural components. The advantages of FGMs are that the materials

\footnotetext{
* Corresponding author.

0013-7944/99/\$ - see front matter (C) 1999 Elsevier Science Ltd. All rights reserved.

PII: S0013-7944(99)00045-4
} 
(a)
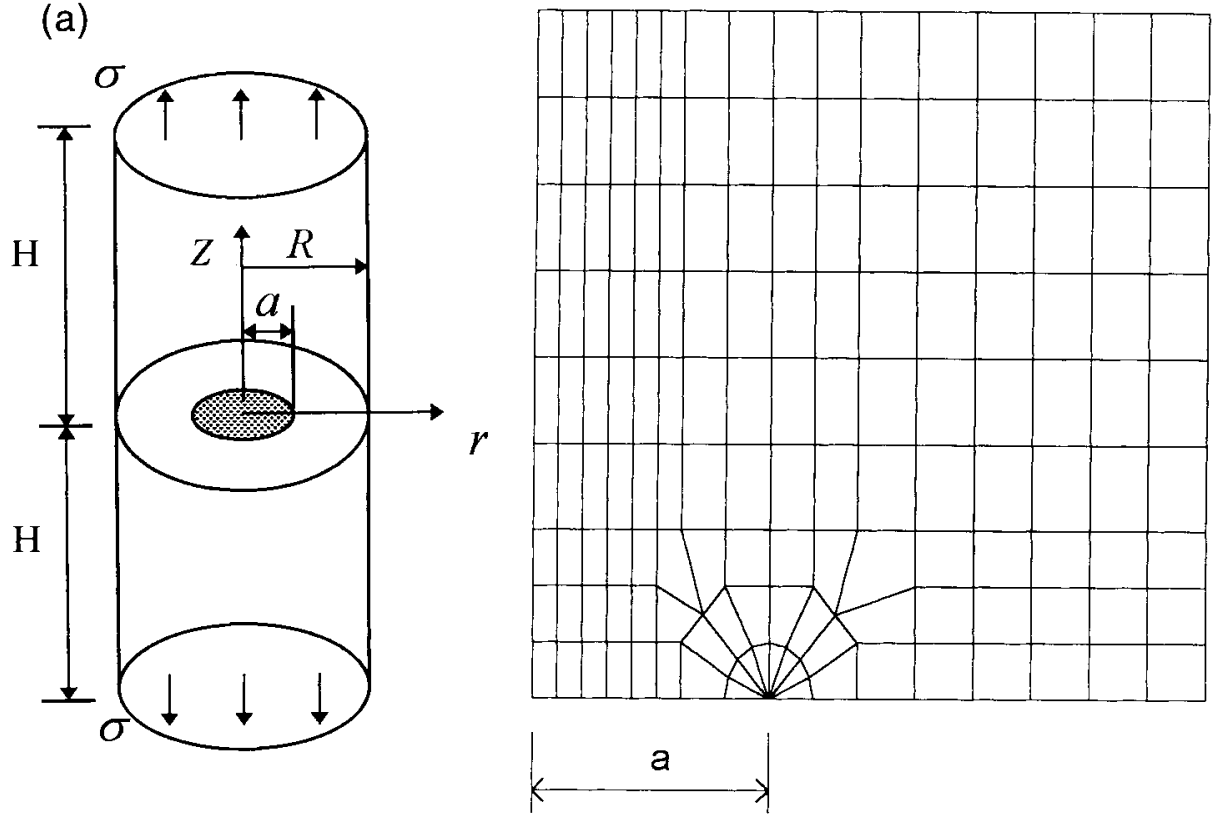

(b)
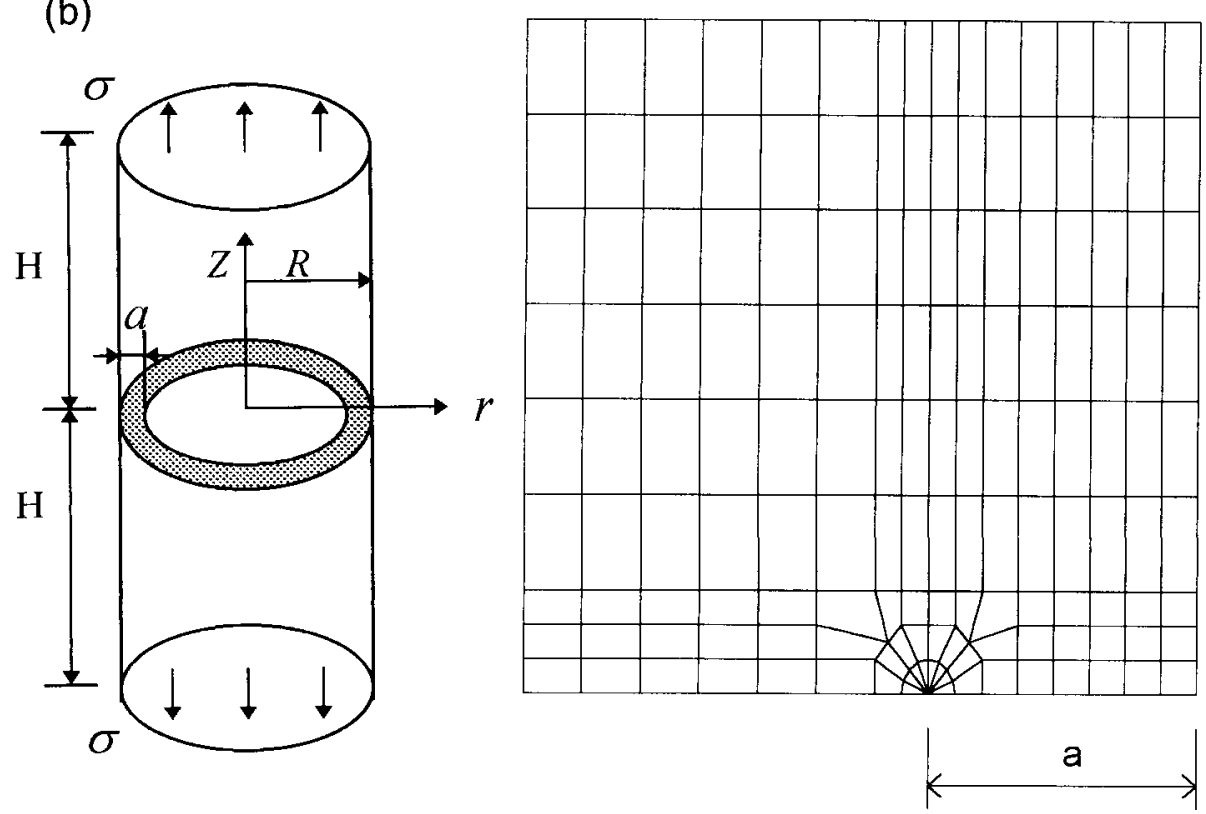

Fig. 1. (a) Cylinder with an embedded penny-shaped crack and its finite element mesh; (b) cylinder with an external circumferential crack and its finite element mesh. 
could resist corrosion, radiation and high temperatures effectively and, at the same time, the residual and thermal stresses in the materials could be relaxed significantly [1]. The interest in FGMs research is growing rapidly due to these advantages. It is expected that FGMs have promising applications in aerospace engineering, chemical engineering and nuclear power plants. From the viewpoints of applied mechanics, FGMs are nonhomogeneous solids. Due to the complexity of the problem, there are only a few papers that have studied the crack problems in FGMs. Erdogan and Delale et al. [2-5] studied some problems of nonhomogeneous elastic materials with cracks. Zou and Wang et al. [6,7] studied some crack problems in composites with nonhomogeneous interlayer. An important conclusion given by these researches is that, the nature of the stress singularity at a crack tip in nonhomogeneous materials would remain to be the standard square-root type as homogeneous solids, provided that the spatial distribution of the material property is continuous near and at the crack tip. Hassan [8] treated the torsion of a nonhomogeneous cylinder slackened by a circular cut. He assumed that the shear modulus was described by a power-law dependence on the radial distance.

However, the analytical approach used by these literature can only deal with some simple distribution of material properties, such as an exponential form [2-5] or power form [6-8], which may be quite different from the real distribution. It is difficult by using the analytical methods to investigate the influences of the actual material property distributions on the fracture mechanics parameters. But, yet these influences are very important knowledge for the optimal design of FGMs. Therefore, numerical methods have to be developed for the analyses of a large range of practical problems.

In numerical methods, the most versatile method is the Finite Element Method (FEM) and it has been extensively used for computational fracture mechanics studies. However, most of these finite element approaches mainly concentrated on homogeneous materials or piecewise homogeneous materials. The finite element formulation suitable for nonhomogeneous materials with continuously varying properties was proposed recently by Li and Zou [9]. This improved FEM has been verified to be very effective and quite efficient for the stress analysis of FGMs.

In this paper, we use the improved FEM to analyze solid cylinders with functionally graded properties. The solid cylinders are assumed to be subjected to remote uniform tension. Two cases of cracks are considered. One is a circumferentially part-through crack at the outer surface of a cylinder. The other is a penny-shaped crack at the center of a cylinder. Our main objective is to investigate the influence of the material property distribution along the cylinder radius on the stress intensity factors.

\section{Material property model}

Consider an FGM solid cylinder described in Fig. 1. It is of radius $R$ and length $2 H$ ( $z$ direction). Assume that the cylinder is fully ceramic at $r=R$ and gradually changes to fully metal at $r=0$, the volume fractions of both phases vary along $r$-direction and the composition in any $\theta-z$ cylindrical face is held constant. The local volume fractions in $r$-direction can be represented by the equations 


$$
V_{\mathrm{c}}(r)=\left[\frac{r}{R}\right]^{p}, \quad V_{\mathrm{m}}(r)=1-V_{\mathrm{c}}(r)
$$

where $V_{\mathrm{m}}$ is the volume fraction of metal, $V_{\mathrm{c}}$ is the volume fraction of ceramic and $p$ is called composition gradient exponent. Using Eq. (1), a great range of FGMs composition profiles can be examined by varying the exponent $p$, as shown in Fig. 2.

Due to the changes in relative proportions of ceramic and metal, the material mechanical properties vary along the radial direction of the solid cylinder. The elastic modulus $E$ and the Poisson's ratio $v$ can be considered to vary with volume fraction as following [10]

$$
\begin{aligned}
& E(r)=\frac{\left(E_{\mathrm{c}} E_{\mathrm{m}}-E_{\mathrm{m}}^{2}\right)\left(1-V_{\mathrm{c}}^{1 / 3}+V_{\mathrm{c}}\right)+E_{\mathrm{m}}^{2}}{E_{\mathrm{c}}+\left(E_{\mathrm{m}}-E_{\mathrm{c}}\right) V_{\mathrm{c}}^{1 / 3}} \\
& v(r)=v_{\mathrm{m}} V_{\mathrm{m}}+v_{\mathrm{c}} V_{\mathrm{c}}
\end{aligned}
$$

where the subscripts $\mathrm{m}$ and $\mathrm{c}$ refer to the metal and ceramic, respectively. The Eq. (2) was found to predict the elastic moduli of two phase composites with a better accuracy than other models [11]. Some variations of the elastic modulus $E$ with volume fractions are illustrated in Fig. 3.

\section{Finite element model}

The displacement finite element method is usually applied to stress analysis problems. Its formulation can be derived by the principle of minimum potential energy [12]. The element stiffness matrix $[K]^{e}$ for axisymmetric problem can be derived as

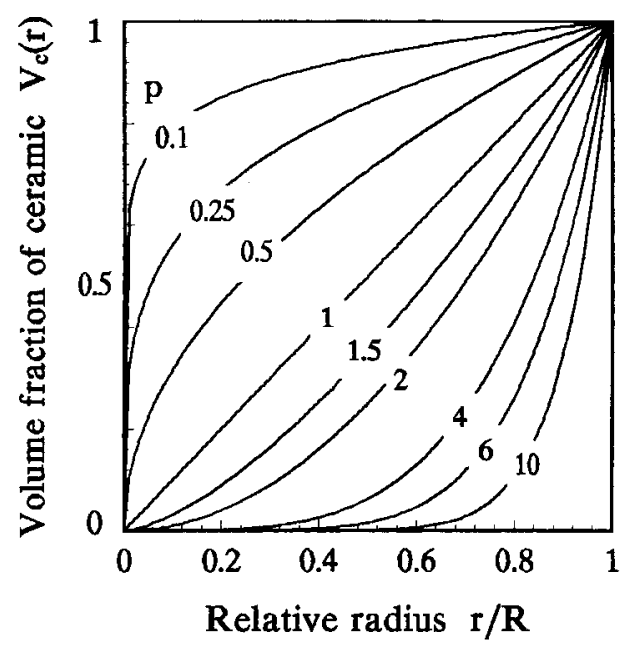

Fig. 2. The composition profiles of ceramic in FGMs. 

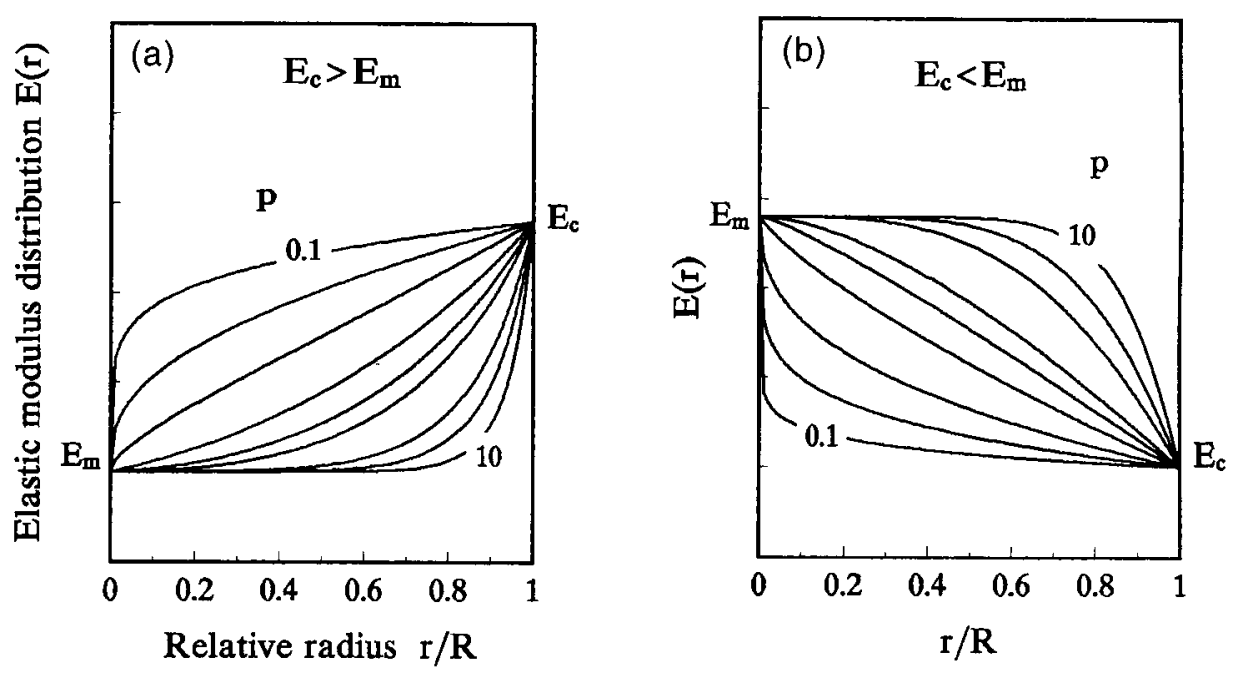

Fig. 3. The distribution of the elastic modulus in FGMs.

$$
[K]^{e}=\iint_{A_{e}}[B]^{\mathrm{T}}[D][B] r \mathrm{~d} r \mathrm{~d} z
$$

where $[B]$ is the strain matrix, $[D]$ is the stress-strain constitutive matrix. In above formulation, only the matrix $[D]$ has relations with the material properties. For elastic isotropic materials under axisymmetric condition, $[D]$ may be expressed as:

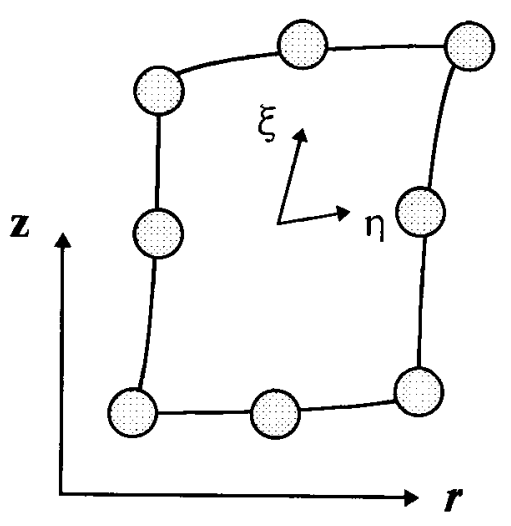

Fig. 4. $m$-Node isoparametric element. 


$$
[D]=\frac{E(1-v)}{(1+v)(1-2 v)}\left[\begin{array}{llll}
1 & \multicolumn{5}{c}{\text { sym. }} \\
\frac{v}{1-v} & 1 & & \\
\frac{v}{1-v} & \frac{v}{1-v} & 1 & \\
0 & 0 & 0 & \frac{1-2 v}{2(1-v)}
\end{array}\right]
$$

For homogeneous materials, $E$ and $v$ are constants. Now for nonhomogeneous FGMs; however, $E$ and $v$ are functions of the $r$ coordinate, as expressed in Eqs. (2) and (3). Therefore, the variations of the material properties must be considered in the finite element analysis for FGMs.

In the paper [9], we adopted the concept of the well-known isoparametric transformation for properly describing the variations of the material properties and then proposed the multiple isoparametric finite element method. Here, we briefly describe it for axisymmetric problems.

Consider a $m$-node axisymmetric element as shown in Fig. 4. The global coordinates of a point on the element at $(\xi, \eta)$ are given by

$$
r=\sum_{i=1}^{m} N_{i}(\xi, \eta) r_{i}, \quad z=\sum_{i=1}^{m} N_{i}(\xi, \eta) z_{i}
$$

where $N_{i}$ are the shape functions corresponding to the node $i$, whose coordinates are $\left(r_{i}, z_{i}\right)$ in the global system and $\left(\xi_{i}, \eta_{i}\right)$ in the local system. As an isoparametric element, the displacements within the element are interpolated as follows:

$$
u=\sum_{i=1}^{m} N_{i}(\xi, \eta) u_{i}, \quad w=\sum_{i=1}^{m} N_{i}(\xi, \eta) w_{i}
$$

where $\left(u_{i}, w_{i}\right)$ are the nodal displacements in the $r$ and $z$ directions. Now, let the material properties $E$ and $v$ at the point $(\xi, \eta)$ be expressed as

$$
\begin{aligned}
& E=\sum_{i=1}^{m} N_{i}(\xi, \eta) E_{i} \\
& v=\sum_{i=1}^{m} N_{i}(\xi, \eta) v_{i}
\end{aligned}
$$

where $\left(E_{i}, v_{i}\right)$ stand for the material properties at the node $i$ of the element. By using Eq. (8), the actual variations of the material properties in a finite element can be approximated by polynomial forms. The degree of the polynomial depends on the number of nodes in the element.

Substituting Eq. (8) into Eq. (4), we obtain the elemental elastic matrix $[D]^{e}$, which becomes a function of the intrinsic coordinates. Then we can calculate the elemental stiffness matrix by the standard Gaussian numerical quadrature in the intrinsic coordinates domain, that is 


$$
[K]^{e}=\int_{-1}^{1} \int_{-1}^{1}[B]^{\mathrm{T}}[D]^{e}[B] r \operatorname{det} \mathbf{J} \mathrm{d} \xi \mathrm{d} \eta
$$

After the elemental stiffness matrices $[K]^{e}$ and the contribution of the nodal force vectors are combined to form a global stiffness matrix $[K]$ and a generalized nodal force vector $\{F\}$ respectively, the vector of the global nodal displacements can be obtained by solving the following global stiffness equations:

$$
[K]\{Q\}=\{F\}
$$

where $\{Q\}$ is the vector of the global nodal displacements. The stresses at any point on a specified element can be obtained by the following equation:

$$
\{\sigma\}=[D]^{e}\{\varepsilon\}=[D]^{e}[B]\{q\}
$$

where $\{q\}$ stands for the nodal displacements of the specified element.

\section{Stress intensity factors computation}

The studies of the literature [2-7] have shown that in nonhomogeneous materials with continuously varying properties, the nature of the stress singularity at a crack tip would remain to be identical to that in homogeneous solids. Jin and Noda [13] have given that the singular terms of the stresses near the crack tip are of the form:

$$
\sigma_{i j}=\frac{K_{\mathrm{I}}}{\sqrt{2 \pi r}} \bar{\sigma}_{i j}^{\mathrm{I}}(\theta)+\frac{K_{\mathrm{II}}}{\sqrt{2 \pi r}} \bar{\sigma}_{i j}^{\mathrm{II}}(\theta)+\frac{K_{\mathrm{III}}}{\sqrt{2 \pi r}} \bar{\sigma}_{i j}^{\mathrm{III}}(\theta)
$$

where $i, j=1,2,3$ stand for the three directions of the spatial coordinate system; $r$ and $\theta$ are the polar coordinates at crack tip. The dimensionless angular functions $\bar{\sigma}_{i j}^{\mathrm{I}}(\theta), \bar{\sigma}_{i j}^{\mathrm{II}}(\theta)$ and $\bar{\sigma}_{i j}^{\mathrm{III}}(\theta)$ are the same as those for homogeneous materials. It has been confirmed (detailed discussion can be found in $\mathrm{Gu}$ and Asaro [14]) that the result is independent of the form for material properties and the orientation of the crack. The stress intensity factors $K_{\mathrm{I}}, K_{\mathrm{II}}$ and $K_{\mathrm{III}}$ are functions of the material gradients, external loading and geometry. Material gradients do not affect the order of the singularity and the angular functions, but do affect the stress intensity factors.

The importance of the above results lies in the fact that in FGMs one can now use the crack tip finite element modeling developed for the ordinary square-root singularity and the computational methods for the SIFs in homogeneous solids can be adopted for that in nonhomogeneous solids with a few modifications.

There are many ways of evaluating the SIFs from finite element solution. These include the extrapolation of displacement and/or stress fields to the crack tip; Rice's contour integral J; the strain energy approach; the virtual crack extension technique, and so on. In this paper, we use the displacement extrapolation technique.

The so-called triangular quarter-point elements $[15,16]$ are used as crack tip elements. It has been verified that this kind of element results in a $1 / \sqrt{r}$ strain singularity within the elements 
as well as on the element edges. The SIFs are obtained by two-point formula. The procedure is briefly described as following:

Consider a crack tip region shown in Fig. 5. The displacement fields on the crack surfaces can be written as

$$
\begin{aligned}
& w(\theta=\pi)-w(\theta=-\pi)=\frac{\kappa+1}{\mu} K_{\mathrm{I}} \sqrt{\frac{r}{2 \pi}} \\
& u(\theta=\pi)-u(\theta=-\pi)=\frac{\kappa+1}{\mu} K_{\mathrm{II}} \sqrt{\frac{r}{2 \pi}}
\end{aligned}
$$

where $\kappa=(3-4 v)$ for plane strain and $\kappa=(3-v) /(1+v)$ for plane stress, $\mu=E / 2(1+v)$ is the shear modulus. For the points $d$ and $e$ with $r=L$, we obtain

$$
\begin{aligned}
& K_{\mathrm{I}}(L)=\sqrt{\frac{2 \pi}{L}} \frac{\mu(L)}{\kappa(L)+1}\left(w_{d}-w_{e}\right) \\
& K_{\mathrm{II}}(L)=\sqrt{\frac{2 \pi}{L}} \frac{\mu(L)}{\kappa(L)+1}\left(u_{d}-u_{e}\right)
\end{aligned}
$$

For the points $b$ and $c$ with $r=L / 4$, we obtain

$$
K_{\mathrm{I}}(L / 4)=\sqrt{\frac{2 \pi}{L}} \frac{2 \mu(L / 4)}{\kappa(L / 4)+1}\left(w_{b}-w_{c}\right)
$$

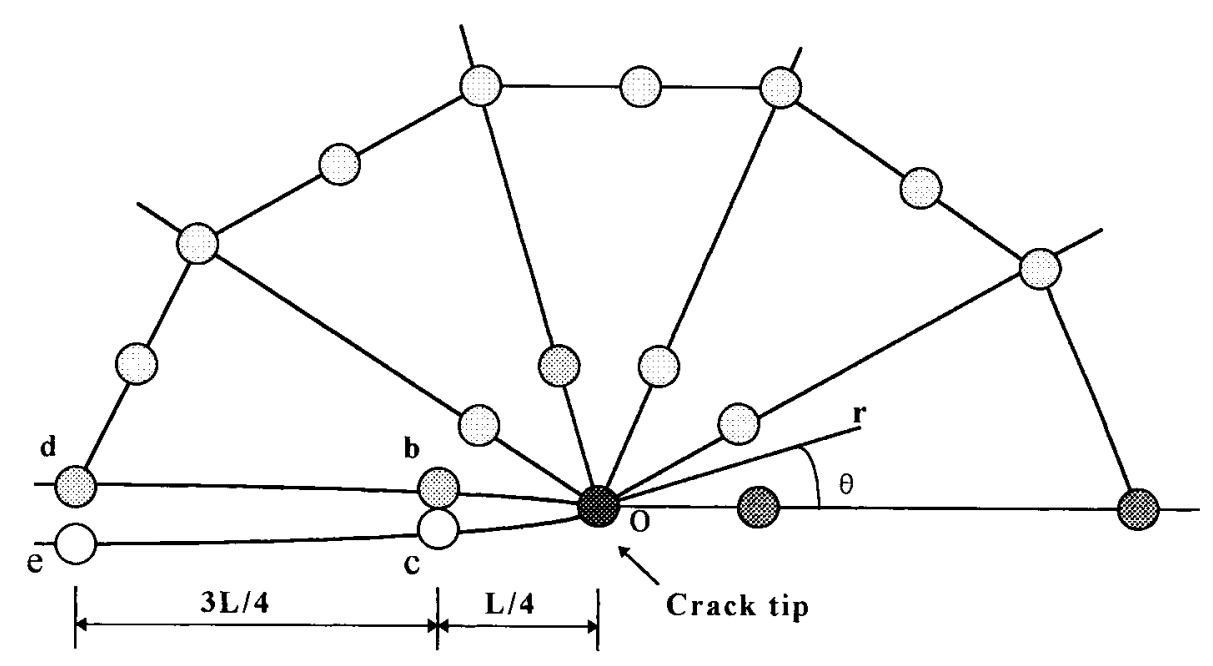

Fig. 5. Triangular quarter-point elements around the crack-tip. 


$$
K_{\mathrm{II}}(L / 4)=\sqrt{\frac{2 \pi}{L}} \frac{2 \mu(L / 4)}{\kappa(L / 4)+1}\left(u_{b}-u_{c}\right)
$$

Then, the values of $K_{\mathrm{I}}$ and $K_{\mathrm{II}}$ at the tip $o$ are obtained by linear extrapolation as following [17]:

$$
\begin{aligned}
& K_{\mathrm{I}}=2 K_{\mathrm{I}}(L / 4)-K_{\mathrm{I}}(L) \\
& K_{\mathrm{II}}=2 K_{\mathrm{II}}(L / 4)-K_{\mathrm{II}}(L)
\end{aligned}
$$

It should be emphasized that the values of the material properties in the above equations are the values of the corresponding points.

\section{Numerical results and discussion}

As shown in Fig. 1, the crack plane is a plane of symmetry and the crack problem is one of mode I. Because of the symmetry, we only consider the $z>0$ part of the cylinder. The finite element division in the axisymmetric face is shown in Fig. 1. The crack tip elements are sixnode triangular quarter-point elements. Other elements are eight-node quadrilateral elements. The lengths of all element sides emanating from the crack tip are selected as $1 / 10$ of the crack length for $a / t \leq 0.7$, and $1 / 20$ for $a / t=0.8$. This selection is made from the error analyses of the computed results for edge cracked plate, which has been given analytical solution by Erdogan [5]. In that case, the relative errors for all crack-depths and all values of $E_{\mathrm{c}} / E_{\mathrm{m}}$ were less than $\pm 3 \%$.

It should be mentioned that the mid-side nodes on the crack tip elements remain at the midside position for simulating the variations of the material properties in the crack tip elements.

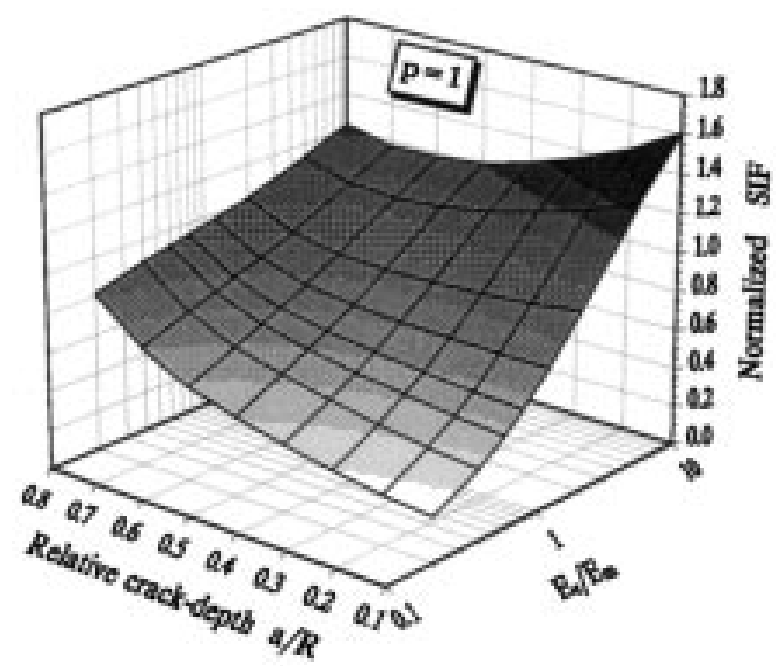

Fig. 6. The effect of $E_{\mathrm{c}} / E_{\mathrm{m}}$ on the normalized stress intensity factors (for embedded penny-shaped crack). 
The normalized mode I stress intensity factor is defined by

$$
F_{\mathrm{I}}=\frac{K_{\mathrm{I}}}{\sigma \sqrt{\pi a}}
$$

In this study, we mainly investigate the influences of $E(r)$ on the normalized SIFs. The Poisson's ratio is assumed to be constant $(v=0.3)$. The ratio of $E_{\mathrm{c}} / E_{\mathrm{m}}$ is assumed as $0.2,0.4$, $0.6,0.8,1.2,2.0,5.0$ and 10.0, respectively. The material gradient exponent $p$ is selected as 0.1 , $0.25,0.5,1.0,1.5,2.0,4.0,6.0$ and 10.0 , respectively. The ratio $H / R$ is taken as 5.0.
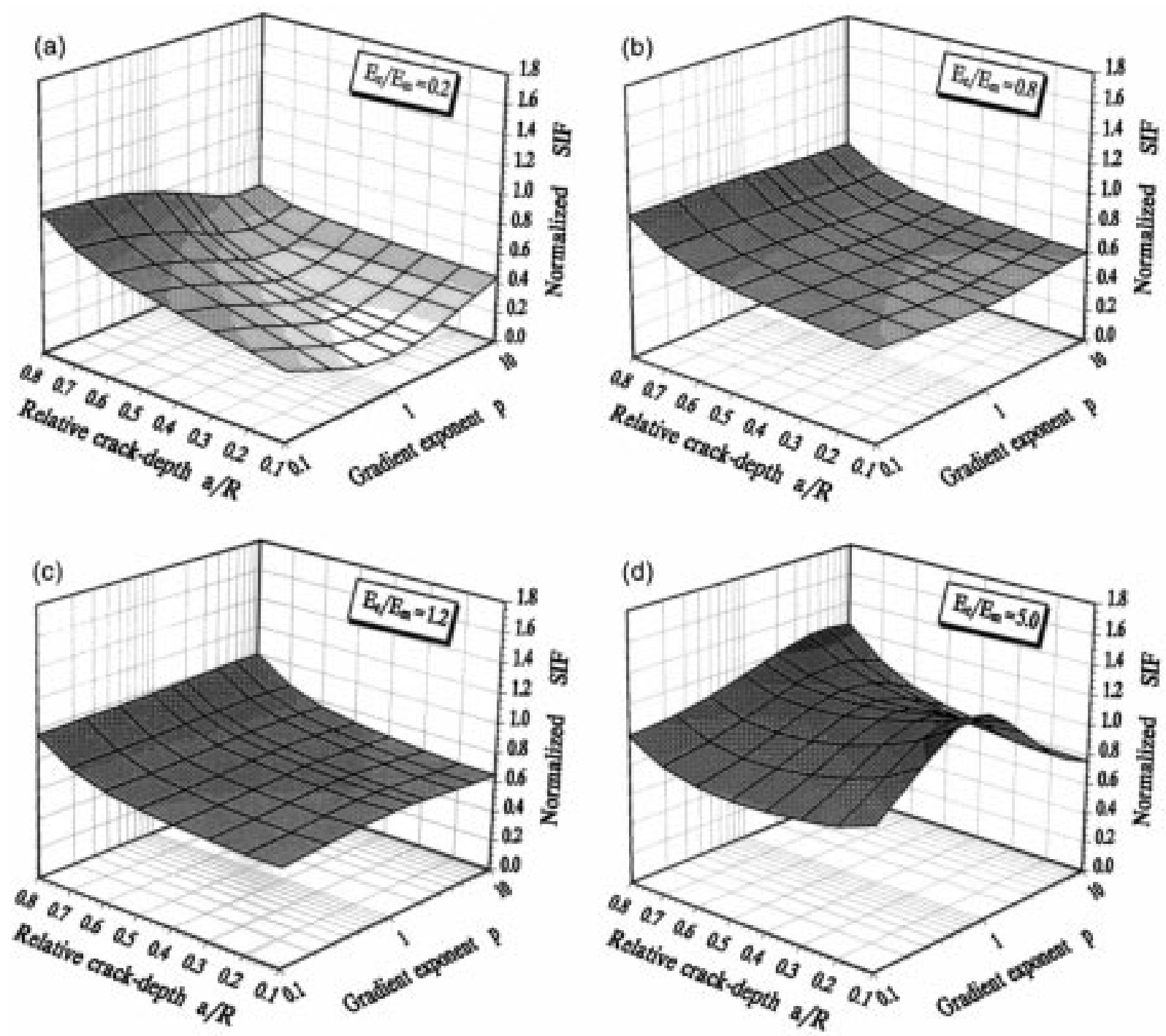

Fig. 7. The effect of compositional gradient on the normalized stress intensity factors (for embedded penny-shaped crack). 


\subsection{Embedded penny-shaped cracks}

Some normalized SIFs for embedded penny-shaped cracks are shown in Figs. 6,7 and Table 1.

Fig. 6 shows the effect of different couple of FGM phases on the normalized SIFs when the

Table 1

The normalized stress intensity factors for FGM solid cylinders with a penny-shaped crack

\begin{tabular}{|c|c|c|c|c|c|c|c|c|c|}
\hline \multirow[t]{2}{*}{$a / R$} & \multicolumn{9}{|l|}{$p$} \\
\hline & 0.10 & 0.25 & 0.50 & 1.00 & 1.50 & 2.00 & 4.00 & 6.00 & 10.00 \\
\hline \multicolumn{10}{|c|}{$E_{\mathrm{c}} / E_{\mathrm{m}}=0.4$} \\
\hline 0.1 & 0.5368 & 0.4512 & 0.3843 & 0.3569 & 0.3691 & 0.3880 & 0.4489 & 0.4856 & 0.5267 \\
\hline 0.2 & 0.5669 & 0.4964 & 0.4294 & 0.3836 & 0.3824 & 0.3946 & 0.4511 & 0.4882 & 0.5302 \\
\hline 0.3 & 0.5999 & 0.5418 & 0.4791 & 0.4226 & 0.4091 & 0.4127 & 0.4604 & 0.4986 & 0.5425 \\
\hline 0.4 & 0.6295 & 0.5828 & 0.5270 & 0.4665 & 0.4429 & 0.4376 & 0.4698 & 0.5070 & 0.5536 \\
\hline 0.5 & 0.6661 & 0.6294 & 0.5817 & 0.5220 & 0.4912 & 0.4773 & 0.4880 & 0.5208 & 0.5695 \\
\hline 0.6 & 0.7112 & 0.6826 & 0.6425 & 0.5867 & 0.5531 & 0.5328 & 0.5201 & 0.5428 & 0.5902 \\
\hline 0.7 & 0.7970 & 0.7761 & 0.7455 & 0.6974 & 0.6628 & 0.6379 & 0.5976 & 0.6009 & 0.6368 \\
\hline 0.8 & 0.9519 & 0.9387 & 0.9183 & 0.8814 & 0.8529 & 0.8260 & 0.7645 & 0.7388 & 0.7398 \\
\hline \multicolumn{10}{|c|}{$E_{\mathrm{c}} / E_{\mathrm{m}}=0.6$} \\
\hline 0.1 & 0.5775 & 0.5252 & 0.4811 & 0.4617 & 0.4704 & 0.4838 & 0.5254 & 0.5490 & 0.5740 \\
\hline 0.2 & 0.5969 & 0.5552 & 0.5131 & 0.4827 & 0.4820 & 0.4903 & 0.5284 & 0.5524 & 0.5778 \\
\hline 0.3 & 0.6228 & 0.5892 & 0.5511 & 0.5153 & 0.5064 & 0.5089 & 0.5406 & 0.5650 & 0.5918 \\
\hline 0.4 & 0.6469 & 0.6203 & 0.5875 & 0.5505 & 0.5356 & 0.5322 & 0.5532 & 0.5770 & 0.6053 \\
\hline 0.5 & 0.6793 & 0.6585 & 0.6314 & 0.5958 & 0.5771 & 0.5686 & 0.5753 & 0.5959 & 0.6255 \\
\hline 0.6 & 0.7216 & 0.7053 & 0.6829 & 0.6508 & 0.6308 & 0.6186 & 0.6112 & 0.6251 & 0.6540 \\
\hline 0.7 & 0.8038 & 0.7925 & 0.7753 & 0.7487 & 0.7288 & 0.7144 & 0.6902 & 0.6919 & 0.7144 \\
\hline 0.8 & 0.9551 & 0.9483 & 0.9368 & 0.9177 & 0.9013 & 0.8876 & 0.8520 & 0.8366 & 0.8366 \\
\hline \multicolumn{10}{|c|}{$E_{\mathrm{c}} / E_{\mathrm{m}}=2.0$} \\
\hline 0.1 & 0.7218 & 0.8138 & 0.8916 & 0.9188 & 0.8932 & 0.8606 & 0.7737 & 0.7335 & 0.6965 \\
\hline 0.2 & 0.6996 & 0.7698 & 0.8387 & 0.8830 & 0.8775 & 0.8569 & 0.7802 & 0.7394 & 0.7016 \\
\hline 0.3 & 0.6992 & 0.7543 & 0.8141 & 0.8651 & 0.8728 & 0.8639 & 0.8021 & 0.7608 & 0.7210 \\
\hline 0.4 & 0.7043 & 0.7472 & 0.7974 & 0.8487 & 0.8657 & 0.8660 & 0.8245 & 0.7856 & 0.7429 \\
\hline 0.5 & 0.7217 & 0.7548 & 0.7957 & 0.8439 & 0.8658 & 0.8742 & 0.8555 & 0.8228 & 0.7788 \\
\hline 0.6 & 0.7541 & 0.7798 & 0.8136 & 0.8577 & 0.8819 & 0.8944 & 0.8957 & 0.8734 & 0.8309 \\
\hline 0.7 & 0.8262 & 0.8447 & 0.8696 & 0.9064 & 0.9308 & 0.9476 & 0.9699 & 0.9634 & 0.9325 \\
\hline 0.8 & 0.9685 & 0.9782 & 0.9953 & 1.0222 & 1.0442 & 1.0603 & 1.0984 & 1.1124 & 1.1087 \\
\hline \multicolumn{10}{|c|}{$E_{\mathrm{c}} / E_{\mathrm{m}}=10.0$} \\
\hline 0.1 & 1.1931 & 1.5510 & 1.7041 & 1.6114 & 1.4474 & 1.3074 & 1.0042 & 0.8845 & 0.7852 \\
\hline 0.2 & 1.0338 & 1.3127 & 1.4683 & 1.4632 & 1.3702 & 1.2731 & 1.0140 & 0.8934 & 0.7921 \\
\hline 0.3 & 0.9495 & 1.1740 & 1.3207 & 1.3605 & 1.3139 & 1.2514 & 1.0419 & 0.9237 & 0.8163 \\
\hline 0.4 & 0.8937 & 1.0748 & 1.2080 & 1.2724 & 1.2580 & 1.2223 & 1.0667 & 0.9587 & 0.8460 \\
\hline 0.5 & 0.8643 & 1.0095 & 1.1280 & 1.2057 & 1.2143 & 1.2010 & 1.0993 & 1.0083 & 0.8950 \\
\hline 0.6 & 0.8647 & 0.9848 & 1.0926 & 1.1779 & 1.2018 & 1.2025 & 1.1434 & 1.0730 & 0.9670 \\
\hline 0.7 & 0.9044 & 0.9964 & 1.0879 & 1.1768 & 1.2131 & 1.2294 & 1.2201 & 1.1796 & 1.0969 \\
\hline 0.8 & 1.0159 & 1.0778 & 1.1481 & 1.2327 & 1.2791 & 1.3072 & 1.3469 & 1.3447 & 1.3070 \\
\hline
\end{tabular}


composition gradients are linear. It can be seen that the parameter $E_{\mathrm{c}} / E_{\mathrm{m}}$ has quite considerable influence on the SIFs. The normalized SIFs increase with the increase of $E_{\mathrm{c}} / E_{\mathrm{m}}$ value. The less the ratio of $a / R$, the greater the increasing velocity of the normalized SIF with $E_{\mathrm{c}} / E_{\mathrm{m}}$.

Fig. 7(a)-(d) show the influences of the compositional gradient on the normalized SIFs. It can be seen that the variations of the gradient exponent $p$ have quite obvious influences on the SIFs when the differences between the values of $E_{\mathrm{m}}$ and $E_{\mathrm{c}}$ are relatively greater. However, the influences of $p$ decrease with the reduction of the differences between $E_{\mathrm{m}}$ and $E_{\mathrm{c}}$. Especially, for $a / R \leq 0.5$, when $E_{\mathrm{c}} / E_{\mathrm{m}}<1$, the normalized SIFs for fixed $a / R$ tend the lowest at about $p=1$; when $E_{\mathrm{c}} / E_{\mathrm{m}}>1$, the normalized SIFs for fixed $a / R$ tend the largest at about $p=1$. The same conclusions can also be obtained from Table 1 .

Thus, if on the viewpoint of fracture mechanics, we can say that the optimal composition depends on the couple of FGM phases. No sole optimal composition exists for different FGMs.

\subsection{External circumferential cracks}

Figs. 8 and 9 show some results of the normalized SIFs for external circumferential cracks. Additional results are stipulated in Table 2.

Fig. 8 shows the effect of the value of $E_{\mathrm{c}} / E_{\mathrm{m}}$ on the normalized SIFs when the FGM phases are mixed linearly. It can be seen that the parameter $E_{\mathrm{c}} / E_{\mathrm{m}}$ also has considerable influence on the SIFs. However, the influence is different from that of a penny-shaped crack. The normalized SIFs decrease with the increase of $E_{\mathrm{c}} / E_{\mathrm{m}}$ value.

Fig. 9(a)-(d) show the influences of the compositional gradient on the normalized SIFs. It can be seen that the variations of the gradient exponent $p$ also have obvious influences on the SIFs when the differences between the values of $E_{\mathrm{m}}$ and $E_{\mathrm{c}}$ are relatively greater. But the

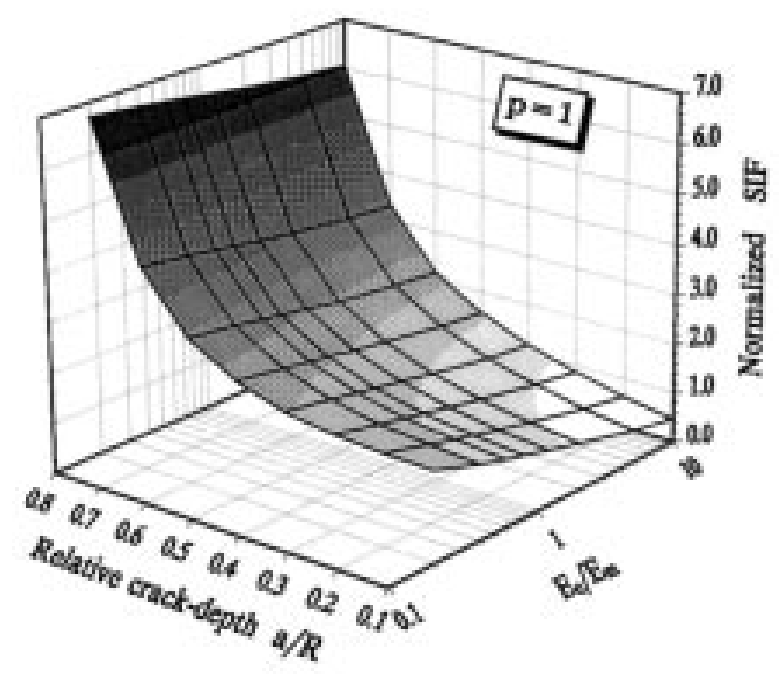

Fig. 8. The effect of $E_{\mathrm{c}} / E_{\mathrm{m}}$ on the normalized stress intensity factors (for external circumferential crack). 

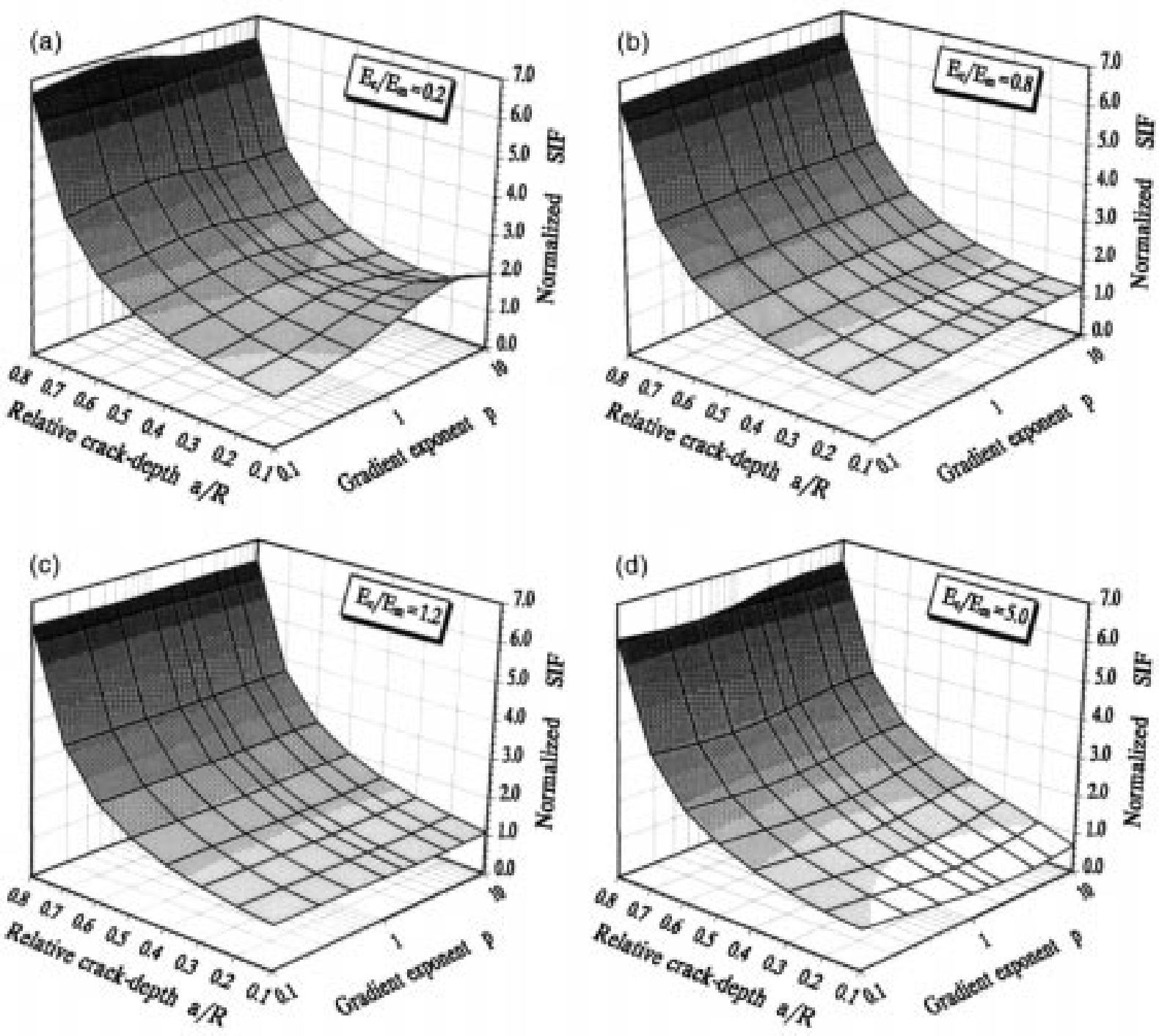

Fig. 9. The effect of compositional gradient on the normalized stress intensity factors (for external circumferential crack).

influence in this case is weaker than that in the case of a penny-shaped crack. Similarly, the influences of $p$ decrease with the reduction of the differences between $E_{\mathrm{m}}$ and $E_{\mathrm{c}}$. When the value of $E_{\mathrm{c}} / E_{\mathrm{m}}$ is between 0.8 and 1.2 , the influence of $p$ can be negligible. Also, the optimal composition depends on the couple of FGM phases.

\section{Conclusion}

The FGM solid cylinders with an external circumferential crack or an embedded penny- 
Table 2

The normalized stress intensity factors for FGM solid cylinders with an external circumferential crack

\begin{tabular}{|c|c|c|c|c|c|c|c|c|c|}
\hline \multirow[t]{2}{*}{$a / R$} & \multicolumn{9}{|l|}{$p$} \\
\hline & 0.10 & 0.25 & 0.50 & 1.00 & 1.50 & 2.00 & 4.00 & 6.00 & 10.00 \\
\hline \multicolumn{10}{|c|}{$E_{\mathrm{c}} / E_{\mathrm{m}}=0.4$} \\
\hline 0.1 & 1.2033 & 1.2657 & 1.3547 & 1.4838 & 1.5793 & 1.6396 & 1.7296 & 1.7171 & 1.6048 \\
\hline 0.2 & 1.3065 & 1.3668 & 1.4496 & 1.5592 & 1.6218 & 1.6544 & 1.6405 & 1.5647 & 1.4437 \\
\hline 0.3 & 1.4533 & 1.5128 & 1.5865 & 1.6705 & 1.7037 & 1.7083 & 1.6313 & 1.5539 & 1.4809 \\
\hline 0.4 & 1.6737 & 1.7318 & 1.7987 & 1.8608 & 1.8670 & 1.8516 & 1.7517 & 1.6961 & 1.6595 \\
\hline 0.5 & 2.0305 & 2.0901 & 2.1516 & 2.1893 & 2.1728 & 2.1418 & 2.0443 & 2.0121 & 1.9970 \\
\hline 0.6 & 2.6101 & 2.6785 & 2.7397 & 2.7589 & 2.7232 & 2.6779 & 2.5855 & 2.5643 & 2.5569 \\
\hline 0.7 & 3.7701 & 3.8496 & 3.9079 & 3.8903 & 3.8286 & 3.7790 & 3.7053 & 3.6950 & 3.6922 \\
\hline 0.8 & 6.5459 & 6.6546 & 6.7057 & 6.6141 & 6.5223 & 6.4685 & 6.4262 & 6.4222 & 6.4220 \\
\hline \multicolumn{10}{|c|}{$E_{\mathrm{c}} / E_{\mathrm{m}}=0.6$} \\
\hline 0.1 & 1.1845 & 1.2186 & 1.2652 & 1.3326 & 1.3810 & 1.4142 & 1.4648 & 1.4540 & 1.3978 \\
\hline 0.2 & 1.2855 & 1.3190 & 1.3625 & 1.4221 & 1.4575 & 1.4740 & 1.4693 & 1.4311 & 1.3650 \\
\hline 0.3 & 1.4324 & 1.4643 & 1.5049 & 1.5529 & 1.5712 & 1.5746 & 1.5341 & 1.4916 & 1.4496 \\
\hline 0.4 & 1.6523 & 1.6836 & 1.7213 & 1.7564 & 1.7623 & 1.7544 & 1.6996 & 1.6666 & 1.6447 \\
\hline 0.5 & 2.0075 & 2.0400 & 2.0754 & 2.0983 & 2.0908 & 2.0745 & 2.0179 & 1.9977 & 1.9876 \\
\hline 0.6 & 2.5814 & 2.6196 & 2.6550 & 2.6658 & 2.6479 & 2.6247 & 2.5710 & 2.5568 & 2.5518 \\
\hline 0.7 & 3.7322 & 3.7784 & 3.8126 & 3.8044 & 3.7695 & 3.7413 & 3.6982 & 3.6913 & 3.6909 \\
\hline 0.8 & 6.4890 & 6.5514 & 6.5764 & 6.5339 & 6.4832 & 6.4511 & 6.4236 & 6.4224 & 6.4225 \\
\hline \multicolumn{10}{|c|}{$E_{\mathrm{c}} / E_{\mathrm{m}}=2.0$} \\
\hline 0.1 & 1.1165 & 1.0664 & 1.0037 & 0.9278 & 0.8861 & 0.8618 & 0.8357 & 0.8497 & 0.9013 \\
\hline 0.2 & 1.2184 & 1.1696 & 1.1130 & 1.0481 & 1.0189 & 1.0071 & 1.0238 & 1.0638 & 1.1322 \\
\hline 0.3 & 1.3645 & 1.3169 & 1.2656 & 1.2161 & 1.2003 & 1.2014 & 1.2469 & 1.2936 & 1.3491 \\
\hline 0.4 & 1.5819 & 1.5364 & 1.4895 & 1.4534 & 1.4509 & 1.4617 & 1.5227 & 1.5660 & 1.6000 \\
\hline 0.5 & 1.9320 & 1.8856 & 1.8439 & 1.8224 & 1.8317 & 1.8507 & 1.9178 & 1.9482 & 1.9659 \\
\hline 0.6 & 2.4911 & 2.4400 & 2.4014 & 2.3915 & 2.4137 & 2.4392 & 2.5075 & 2.5303 & 2.5404 \\
\hline 0.7 & 3.6158 & 3.5584 & 3.5229 & 3.5355 & 3.5731 & 3.6057 & 3.6670 & 3.6781 & 3.6823 \\
\hline 0.8 & 6.3162 & 6.2409 & 6.2161 & 6.2677 & 6.3258 & 6.3663 & 6.4133 & 6.4181 & 6.4179 \\
\hline \multicolumn{10}{|c|}{$E_{\mathrm{c}} / E_{\mathrm{m}}=10.0$} \\
\hline 0.1 & 0.9102 & 0.7220 & 0.5768 & 0.4711 & 0.4373 & 0.4276 & 0.4559 & 0.5082 & 0.6150 \\
\hline 0.2 & 1.0169 & 0.8430 & 0.7188 & 0.6445 & 0.6345 & 0.6438 & 0.7302 & 0.8195 & 0.9585 \\
\hline 0.3 & 1.1690 & 1.0115 & 0.9107 & 0.8668 & 0.8791 & 0.9057 & 1.0316 & 1.1336 & 1.2585 \\
\hline 0.4 & 1.3893 & 1.2475 & 1.1683 & 1.1536 & 1.1840 & 1.2242 & 1.3720 & 1.4675 & 1.5557 \\
\hline 0.5 & 1.7344 & 1.6061 & 1.5476 & 1.5613 & 1.6095 & 1.6614 & 1.8159 & 1.8934 & 1.9455 \\
\hline 0.6 & 2.2704 & 2.1491 & 2.1080 & 2.1497 & 2.2159 & 2.2780 & 2.4359 & 2.4973 & 2.5282 \\
\hline 0.7 & 3.3586 & 3.2440 & 3.2283 & 3.3105 & 3.4006 & 3.4742 & 3.6265 & 3.6644 & 3.6781 \\
\hline 0.8 & 5.9693 & 5.8605 & 5.8919 & 6.0448 & 6.1737 & 6.2641 & 6.3956 & 6.4152 & 6.4182 \\
\hline
\end{tabular}

shaped crack are studied by using the improved FEM. It is revealed that the material property distribution has quite considerable influence on the SIFs. The differences in stress intensity factors for different FGMs are obvious. The composition of FGMs influences the stress intensity factors only when the difference between the elastic modulus of metal and that of ceramic is relatively larger. The influence of composition gradient for an embedded pennyshaped crack is stronger than that for an external circumferential crack. On the viewpoint of 
fracture mechanics, the optimization of FGM composition is necessary and the optimal composition gradient depends on the type of FGMs.

\section{Acknowledgements}

This research was supported by the Chinese National Natural Science Fund of People's Republic of China under project No. 19772029 and key project No. 19891180.

\section{References}

[1] Koizumi M. The concept of ZGM. In: Holt J, Koizumi M, Hirai T, Munir Z, (eds.). Functionally graded materials, Ceramic Transactions 1993;34;3-10.

[2] Delale F, Erdogan F. The crack problem for a nonhomogeneous plane. Trans ASME/ J Appl Mech 1983;50:609-14.

[3] Erdogan F. The crack problem for bonded nonhomogeneous materials under antiplane shear loading. Trans ASME/ J Appl Mech 1985;52:823-8.

[4] Konda N, Erdogan F. The mixed mode crack problem in a nonhomogeneous elastic medium. Engng Fracture Mech 1994;47:533-45.

[5] Erdogan F, Wu BH. The surface crack problem for a plate with functionally graded properties. Trans ASME/ J Appl Mech 1997;64:449-56.

[6] Zou ZZ, Wang XY, Wang D. On the modeling of interfacial zone containing a Griffith crack: plane problem. Key Engineering Material 1997;145-149:489-94.

[7] Wang Xuyue, Zhenzhu Zou, Wang Duo. On the penny-shaped crack in a nonhomogeneous interlayer of adjoining two different elastic materials. Int J Solids Structures 1997;34:3911-21.

[8] Hassan HAZ. Torsion of a nonhomogeneous infinite elastic cylinder slackened by a circular cut. J Engng Math 1996;30:547-55.

[9] Li Chunyu, Zou Zhenzhu, Multiple isoparametric finite element method for nonhomogeneous media. Mechanics Research Communications, 1999 (in press).

[10] Ravichandran KS. Elastic properties of two phase composites. J Am Ceram Soc 1994;77:1178-84.

[11] Ravichandran KS. Thermal residual stresses in a functionally graded material system. Materials Science and Engineering 1995;A201:269-76.

[12] Zienkiewicz OC, Taylor RL. The finite element method, 3rd. ed. New York: McGraw-Hill, 1977.

[13] Jin ZH, Noda N. Crack tip singularity fields in nonhomogeneous materials. Trans ASME/J Appl Mech 1994;61(3):738-40.

[14] Pei Gu, Asaro RJ. Cracks in functionally graded materials. Int J Solids Structures 1997;34:1-17.

[15] Barsoum RS. On the use of isoparametric finite elements in linear fracture mechanics. Int J Num Meth Engng 1976;10:25-37.

[16] Barsoum RS. Triangular quarter point elements as elastic and perfectly-plastic crack tip elements. Int J Num Meth Engng 1977;11:85-98.

[17] Barsoum RS. Author's reply to the discussion by Tracey. Int J Num Meth Engng 1977;11:402-3. 